PROCEEDINGS OF THE

AMERICAN MATHEMATICAL SOCIETY

Volume 136, Number 10, October 2008, Pages 3539-3548

S 0002-9939(08)09498-7

Article electronically published on June 11, 2008

\title{
THE SERRE DUALITY THEOREM FOR A NON-COMPACT WEIGHTED CR MANIFOLD
}

\author{
MITSUHIRO ITOH, JUN MASAMUNE, AND TAKANARI SAOTOME
}

(Communicated by Mikhail Shubin)

\begin{abstract}
It is proved that the Hodge decomposition and Serre duality hold on a non-compact weighted CR manifold with negligible boundary. A complete CR manifold has negligible boundary. Some examples of complete CR manifolds are presented.
\end{abstract}

\section{INTRODUCTION}

Let $M$ be a strongly pseudo-convex CR manifold, an s.p.c. CR manifold for short, without boundary. A weighted $C R$ manifold is an s.p.c. CR manifold endowed with a measure $\mu$, which has a smooth positive density $\eta$ with respect to the volume form of the CR structure. Then the space $(M, \mu)$ has a natural weighted Kohn Laplacian $\square_{\mu}$, which we call the Witten-Kohn Laplacian.

In this article, we are interested in Serre duality and Hodge decomposition on a non-compact weighted CR manifold. The Serre duality of a compact s.p.c. CR manifold was proved by Tanaka [15] for the case of a trivial line bundle, and recently, the first and the third named authors generalized it to any holomorphic vector bundle $E[8$. On the other hand, Kohn's Hodge decomposition for a compact s.p.c. CR manifold was extended to a general s.p.c. CR manifold with negligible boundary (Definition 2.4) when $E$ is a trivial line bundle by the second author [13.

The aim of the present article is to extend these results to an arbitrary holomorphic vector bundle $E$ over a general weighted $\mathrm{CR}$ manifold with negligible boundary, and to relate them to each other. Namely, by denoting $\mathbb{H}^{p, q}(E)$ the space of $E$-valued $L^{2}$-harmonic forms of $(p, q)$-type, we will show

Main Theorem. Let $M$ be a $(2 n-1)$-dimensional weighted $C R$ manifold with negligible boundary, and let $E$ be a holomorphic vector bundle over $M$. Then the $L^{2}$-Hodge decomposition

$$
L^{2}\left(\Omega^{\bullet}, q(E)\right)=\mathbb{H}^{\bullet}, q \oplus \overline{\operatorname{range}\left(\bar{\partial}^{q-1}\right)} L^{2} \oplus \overline{\text { range }\left(\delta_{\mu}^{q+1}\right)} L^{2}
$$

holds for $0<q<n-1$, and the Serre duality

$$
\sharp_{\mu}: \mathbb{H}^{p, q}(E) \cong \mathbb{H}^{n-p, n-(q+1)}\left(E^{*}\right)
$$

Received by the editors July 17, 2007.

2000 Mathematics Subject Classification. Primary 32V20, 53C17; Secondary 58A14, 14F15.

Key words and phrases. Strongly pseudo-convex manifold, CR manifold, Serre duality, Hodge decomposition, Witten-Kohn Laplacian, weighted Laplacian.

(C)2008 American Mathematical Society Reverts to public domain 28 years from publication 
holds for every $0 \leq p, q \leq n-1$, where $E^{*}$ is the dual bundle of $E$ and $\sharp_{\mu}$ is the complex-conjugate weighted Hodge star operator. In addition, it follows that

$$
\operatorname{ker}\left(\bar{\partial}^{q}\right) / \overline{\operatorname{range}\left(\bar{\partial}^{q-1}\right)} L^{2} \cong \mathbb{H}^{p, q}(E) \stackrel{\sharp_{\mu}}{\cong} \mathbb{H}^{n-p, n-(q+1)}\left(E^{*}\right), \text { for } 0<q<n-1 .
$$

We say that $M$ is complete and $M$ is Riemannian complete if it is complete with respect to the Carnot-Carathéodory distance $d_{C C}$ and Riemannian distance $d_{g}$ associated to the CR structure, respectively (see Section 21). Then we have

Theorem (Proposition 4.1).

(i) If $M$ is Riemannian complete, then it is complete

(ii) If $M$ is complete, then $M$ has negligible boundary.

Therefore, the Main Theorem is applicable, for example, to the Heisenberg group, Sasakian space forms, spherical orbits, and unbranched covering over a compact s.p.c. CR manifold with any weight. These are very important s.p.c. CR manifolds, but they have been excluded from the literature because of their non-compactness (see Section 4). Two main points in the proof are: identification of the weak solution of the Laplace equation $\square_{\mu} \alpha=0$ with the harmonic form (Corollary 2.7); explicit expressions for $\bar{\partial}$ and $\delta_{\mu}$ in terms of $\bar{\partial}_{*}$ and $\delta_{\mu_{*}}$ via $\sharp_{\mu}$ (Lemma 3.3).

We organize the article in the following manner: In Section 2, we recall some necessary notions which we will use in the article. Some new results are given, including the self-adjointness of the $E$-valued Witten-Kohn Laplacian. In Section 3 , we will prove the Serre duality, and finally, in Section 4, we present the examples.

\section{Strongly PSEudo-Convex CR manifolds}

This section contains preliminary results. We recall some definitions related to a strongly pseudo-convex CR manifold $M$, focusing on the construction of the space $\Omega^{p, q}(E)$ of $E$-valued $(p, q)$-forms, its holomorphic structure $\bar{\partial}^{q}$, and the WittenKohn Laplacian $\square_{\mu}$. For a thorough discussion on a geometrical analysis of an s.p.c. CR manifold, we refer the reader to [5] and [15]. We also establish the essential self-adjointness of $\square_{\mu}$ and characterize the harmonic forms as the solutions of the Laplace equation with respect to $\square_{\mu}$. These results are important steps when we extend our knowledge of a compact manifold to a non-compact one.

A $(2 n-1)$-dimensional strongly pseudo-convex $C R$ manifold $M$, we call it simply an s.p.c. CR manifold, is an oriented smooth manifold which carries a structure $(P, J, \theta)$, that is: $P=\operatorname{ker}(\theta) \subset T M$ is an $(n-1)$-dimensional real subbundle with an almost complex structure $J: P \rightarrow P$ satisfying:

$$
[X, Y]-[J X, J Y]-J[J X, Y]-J[X, J Y] \in \Gamma(T M / P), \text { for } X, Y \in \Gamma(P),
$$

and a contact form $\theta \in \Gamma\left((T M / P)^{*}\right)$ whose Levi-form $L(X, Y)=-d \theta(J X, Y)$, for $X, Y \in P$, is positive definite.

Consider the complexification of $J$ and its eigenspace $S=\{X-\sqrt{-1} J X: X \in$ $P\} \subset \mathbb{C} T M$. Then $S \cap \bar{S}=(0)$ and $[\Gamma(S), \Gamma(S)] \subset \Gamma(S)$, where $\bar{S}$ is the complex conjugation of $S$. With the assumption of the strong convexity of $M$, there exist the following implications:

- a Riemannian metric $g=-d \theta+\theta \otimes \theta$;

- a volume form $d v=(n-1) ! \theta \wedge(d \theta)^{n-1}$; 
- a distance $\mathrm{d}_{C C}$ on $M$.

Indeed, since the Levi form $L$ is positive definite, it follows for $0 \neq X \in P_{x}$ that

$$
0 \neq 2 L(X, X)=-(J X(\theta(X))-X(\theta(J X))-\theta([J X, X]))=\theta([J X, X]) .
$$

This shows that $[J X, X] \notin P_{x}$, and hence, $P$ satisfies the Hörmander condition [7. Due to the Chow theorem [4, $P$ implies a non-degenerate distance $\mathrm{d}_{C C}$ on $M$ defined as

$$
\mathrm{d}_{C C}(x, y):=\sup \left\{u(x)-u(y): u \in C^{\infty}(M),\|\pi \nabla u\|_{L^{\infty}} \leq 1\right\},
$$

where $\pi: T M \rightarrow P$ is the projection with respect to $g$. We say $M$ is complete if the distance space $\left(M, \mathrm{~d}_{C C}\right)$ is complete.

Definition 2.1. A complex vector bundle $E$ over $M$ is called holomorphic if it admits a linear differential operator $\bar{\partial}_{E}: \Gamma(E) \rightarrow \Gamma\left(E \otimes \bar{S}^{*}\right)$ satisfying:

(i) $\bar{\partial} \bar{X}(f u)=f \bar{\partial}_{\bar{X}} u+(\bar{X} f) u$;

(ii) $\bar{\partial}_{\bar{X}}\left(\bar{\partial}_{\bar{Y}} u\right)-\bar{\partial}_{\bar{Y}}\left(\bar{\partial}_{\bar{X}} u\right)-\bar{\partial}_{\overline{[X, Y]}} u=0$;

here $f \in C^{\infty}(M), u \in \Gamma(E)$, and $X, Y \in \Gamma(S)$, where $\bar{\partial}_{\bar{X}} u:=\bar{\partial}_{E} u(\bar{X})$.

Example 2.2 (E.g. [15). Let $M$ be a boundary of a strongly pseudoconvex complex manifold and $E$ be a holomorphic vector bundle on the neighbourhood of $M$. Then $\left.E\right|_{M}$ is holomorphic in the above sense.

Hereafter, $E$ stands for a holomorphic vector bundle over $M$. Consider the vector bundle $\hat{T} M=\mathbb{C} T M / \bar{S}$, which is holomorphic (e.g. p. 15 in [15]) together with the operator $\bar{\partial}=\bar{\partial}_{M}$ :

$$
\bar{\partial}_{\bar{X}} u=\varpi([\bar{X}, Z]),
$$

for $u \in \Gamma(\hat{T} M)$ with $Z \in \Gamma(\mathbb{C} T M)$ such that $\varpi(Z)=u$ and $X \in \Gamma(S)$. Here $\varpi: \mathbb{C} T M \rightarrow \hat{T} M$ is the canonical projection. The distinguished vector bundle $E \otimes \bigwedge^{p}(\hat{T} M)^{*}$ with $0 \leq p \leq n-1$ carries a holomorphic structure:

$$
\bar{\partial}_{E} \otimes i d_{\wedge^{p}}+i d_{E} \otimes \bar{\partial}_{\wedge^{p}}
$$

where $i d$ is the identity operator on the indicated space, and $\bigwedge^{p}=\bigwedge^{p} \hat{T} M$. Hereafter we assume additionally that $\left(E, \bar{\partial}_{E}\right)$ is furnished with a smooth Hermitian fiber metric $\langle\cdot, \cdot\rangle_{E}$. The bundle which we will study is

$$
\Omega^{p, q}(E)=\Omega^{p, q}(M ; E)=\Gamma\left(M ; E \otimes \bigwedge^{p} \hat{T} M^{*} \otimes \bigwedge^{q} \bar{S}^{*}\right),
$$

with the holomorphic structure $\bar{\partial}^{q}: \Omega^{p, q}(E) \rightarrow \Omega^{p, q+1}(E)$ defined as

$$
\begin{aligned}
\left(\bar{\partial}^{q} \alpha\right)\left(\bar{X}_{1}, \cdots, \bar{X}_{q+1}\right) & :=\sum(-1)^{i} \bar{\partial}_{\bar{X}_{i}}\left(\alpha\left(\bar{X}_{1}, \cdots, \hat{X}_{i}, \cdots, \bar{X}_{q+1}\right)\right) \\
& +\sum_{i<j}(-1)^{i+j} \alpha\left(\left[\bar{X}_{i}, \bar{X}_{j}\right], \bar{X}_{1}, \cdots, \hat{\bar{X}}_{i}, \cdots, \hat{\bar{X}}_{j}, \cdots, \bar{X}_{q+1}\right),
\end{aligned}
$$

where $\bar{\partial}$ is the holomorphic structure of $E \otimes \bigwedge^{p} \hat{T} M^{*}, \alpha \in \Omega^{p, q}(E)$, and $X_{1}, \cdots, X_{q+1}$ belong to $\Gamma(S)$. If $E$ is the trivial line bundle, we simply denote $\Omega^{p, q}(M)=$ $\Omega^{p, q}(M ; \mathbb{C})$. Set

$$
\begin{gathered}
\Omega^{\bullet} \cdot q(E)=\bigoplus_{q} \Omega^{p, q}(E), \Omega(E):=\bigoplus_{q} \Omega^{\bullet, q}(E) ; \\
\Omega_{0}^{p, q}(E)=\left\{\alpha \in \Omega^{p, q}(E) \mid \alpha \text { has compact support }\right\} .
\end{gathered}
$$


Let $\eta$ be the weight, which is a positive smooth function on $M$, and consider the measure $d \mu=\eta d v$. The associated inner product $(\alpha, \beta)$ of $\alpha, \beta \in \Omega_{0}^{p, q}(E)$ is

$$
(\alpha, \beta)=\int_{M}\langle\alpha, \beta\rangle(x) d \mu(x),
$$

where $\langle\alpha, \beta\rangle(x)$ is the pointwise inner product of $\alpha$ and $\beta$ at $x \in M$. Denote by $\|\alpha\|_{2}$ the norm $\sqrt{(\alpha, \alpha)}$, and by $L^{2}\left(\Omega^{p, q}(E)\right)=L^{2}\left(\Omega^{p, q}(E), \mu\right)$ the set of square integrable $E$-valued measurable $(p, q)$-forms, which coincides with the completion of $\Omega_{0}^{p, q}(E)$ with respect to $\|\cdot\|_{2}$.

Let $\delta_{\mu}^{q}: \Omega^{\bullet}, q+1(E) \rightarrow \Omega^{\bullet}, q(E)$ be the formal adjoint of $\bar{\partial}^{q}$ in $L^{2}(\Omega(E))$. The Witten-Kohn Laplacian $\square_{\mu}^{q}: \Omega^{\bullet}, q(E) \rightarrow \Omega^{\bullet}, q(E)$ is defined by

$$
\square_{\mu}^{q}:=\bar{\partial}^{q-1} \delta_{\mu}^{q-1}+\delta_{\mu}^{q} \bar{\partial}^{q} .
$$

In abbreviation, we remove the super index $q$ when the operator is acting on the space of mixed degree forms. The operator $\square_{\mu}^{q}$ is called subelliptic (e.g. [5], [15]) if there are positive numbers $\sigma$ and $C_{\sigma}$ such that

$$
\|\alpha\|_{(\sigma)}^{2} \leq C_{\sigma}\left(\left(\square_{\mu}^{q} \alpha, \alpha\right)+\|\alpha\|_{2}^{2}\right), \text { for every } \alpha \in \Omega_{0}^{\bullet, q}(E),
$$

where $\|\cdot\|_{(\sigma)}$ is the Sobolev norm of order $\sigma$.

Proposition 2.3. The Witten-Kohn Laplacian $\square_{\mu}^{q}$ is subelliptic for $0<q<n-1$.

Proof. By Lemma 3.3 the Kohn Laplacian $\square=\square_{d v}$, which is the Witten-Kohn Laplacian with $\eta \equiv 1$, has the form:

$$
\square=\sharp^{*} \bar{\partial}_{*} \delta_{*} \sharp+\sharp^{*} \delta_{*} \bar{\partial}_{*} \sharp,
$$

where $\sharp=\sharp_{d v}$ (see Definition 3.1), $\bar{\partial}_{*}$ is the holomorphic structure of $\Omega\left(E^{*}\right)$ and $\delta_{*}$ is its adjoint operator in $L^{2}\left(\Omega\left(E^{*}\right), d v\right)$. Again by Lemma 3.3 and (2.3),

$$
\begin{aligned}
\square_{\mu} \alpha= & \sharp^{*} \bar{\partial}_{* \sharp \sharp^{*}} \delta_{* \sharp \alpha} \alpha \eta^{-1} \sharp^{*}\left(\bar{\partial}_{*} \eta \wedge \sharp \sharp^{*} \delta_{*} \sharp \alpha\right) \\
& +\sharp^{*} \delta_{*} \sharp \sharp^{*} \bar{\partial}_{* \sharp \alpha}+\sharp^{*} \delta_{*} \sharp\left(\eta^{-1} \sharp^{*}\left(\bar{\partial}_{*} \eta \wedge \sharp \alpha\right)\right) \\
= & \square \alpha+\eta^{-1} \sharp^{*}\left(\bar{\partial}_{*} \eta \wedge \delta_{*} \sharp \alpha\right)+\sharp^{*} \delta_{*}\left(\eta^{-1}\left(\bar{\partial}_{*} \eta \wedge \sharp \alpha\right)\right),
\end{aligned}
$$

for $\alpha \in \Omega(E)$. Therefore, $\square_{\mu}$ and $\square$ have the same principal symbols. Since $\square$ is subelliptic [10, we can draw this conclusion.

We consider the following domains:

$$
\begin{gathered}
D\left(\bar{\partial}^{q}\right)=\left\{\alpha \in \Omega^{\bullet, q}: \alpha \text { and } \bar{\partial}^{q} \alpha \text { are square integrable }\right\} ; \\
D\left(\delta_{\mu}^{q}\right)=\left\{\alpha \in \Omega^{\bullet, q+1}: \alpha \text { and } \delta_{\mu}^{q} \alpha \text { are square integrable }\right\} ; \\
D\left(\square_{\mu}^{q}\right)=\left\{\alpha \in D\left(\bar{\partial}^{q}\right) \cap D\left(\delta_{\mu}^{q+1}\right): \bar{\partial} \alpha \in D\left(\delta_{\mu}^{q}\right) \text { and } \delta_{\mu}^{q-1} \alpha \in D\left(\bar{\partial}^{q-1}\right)\right\} .
\end{gathered}
$$

We need the following assumption so that $\square_{\mu}$ is symmetric:

Definition 2.4. We say $M$ has negligible boundary if

$$
(\bar{\partial} \alpha, \beta)=\left(\alpha, \delta_{\mu} \beta\right) \text {, for every } \alpha \in D(\bar{\partial}) \text { and } \beta \in D\left(\delta_{\mu}\right) .
$$

We say $\square_{\mu}^{q}$ is essentially self-adjoint if its $L^{2}$-closure is self-adjoint, and $\square_{\mu}^{q}$ is hypoelliptic if, whenever the distribution $\square_{\mu}^{q} \alpha$ is smooth, then $\alpha$ is smooth.

It is proved in [1] that

Lemma 2.5. A subelliptic operator is hypoelliptic. 
The assumption such that $M$ has negligible boundary implies a stronger property to $\square_{\mu}$ :

Proposition 2.6 (e.g. [13]). If $M$ has negligible boundary, then $\square_{\mu}^{q}$ is essentially self-adjoint in $L^{2}\left(\Omega^{\bullet}, q(E)\right)$ with $0<q<n-1$.

Outline of the proof. Set $\alpha_{\epsilon}=e^{-\square_{\mu} \epsilon} \alpha$ for $\alpha \in D\left({\overline{\delta_{\mu}}}^{L^{2}}\right)$. By Proposition 2.3 and Lemma 2.5. $\alpha_{\epsilon}$ is smooth for every $\epsilon>0$ (here we need the assumption: $0<q<$ $n-1)$. Therefore, since $\bar{\delta}_{\mu}^{L^{2}} \alpha_{\epsilon}=\bar{\partial}^{*} \alpha_{\epsilon} \rightarrow \bar{\partial}^{*} \alpha$ as $\epsilon \rightarrow 0$, we deduce that $\bar{\delta}_{\mu}{ }^{2} \subset \bar{\partial}^{*}$. Since $M$ has negligible boundary, $\bar{\delta}_{\mu}^{L^{2}}=\bar{\partial}^{*}$, and by von Neumann's theorem (e.g. [14]), $\bar{\partial}^{*} \delta_{\mu}^{*}$ is self-adjoint. Moreover, it follows that

$$
\bar{\square}_{\mu}^{L^{2}}=\bar{\partial}^{*} \delta_{\mu}^{*}+\delta_{\mu}^{*} \bar{\partial}^{*},
$$

where the right-hand side is self-adjoint.

We say $\alpha$ is harmonic if $\bar{\partial} \alpha=0$ and $\delta_{\mu} \alpha=0$ in the weak sense. A harmonic form always solves the Laplace equation $\square_{\mu} \alpha=0$, but in general, the converse does not need to be true. However, it follows that

Corollary 2.7. If $M$ has negligible boundary, then the following conditions are equivalent:

(i) $\bar{\partial} \alpha=0$ and $\delta_{\mu} \alpha=0$ pointwise;

(ii) $\alpha$ is harmonic;

(iii) $\alpha$ solves the Laplace equation;

here, $\alpha \in L^{2}\left(C^{\bullet, q}(E)\right)$ and $0<q<n-1$.

Proof. (i) $\Rightarrow$ (ii) is obvious. (ii) $\Rightarrow$ (iii) If $\alpha$ is harmonic, then, $\left(\alpha, \delta_{\mu} \beta\right)=0$ and $(\alpha, \bar{\partial} \gamma)=0$ for every $\beta \in D\left(\delta_{\mu}\right)$ and $\gamma \in D(\bar{\partial})$. This implies $\left(\alpha, \square_{\mu} \beta\right)=0$ for every $\beta \in D\left(\square_{\mu}\right)$; that is, $\alpha$ is the solution of the Laplace equation.

(iii) $\Rightarrow$ (i) Let $\alpha$ be a solution of the Laplace equation. By Proposition 2.6, there exists a sequence $\alpha_{l} \in D\left(\square_{\mu}\right)$ such that

$$
\alpha_{l} \rightarrow \alpha \text { and } \square_{\mu} \alpha_{l} \rightarrow 0 \text {, as } l \rightarrow \infty \text {. }
$$

Due to the fact that $M$ has negligible boundary,

$$
\left\|\bar{\partial} \alpha_{l}\right\|_{2}^{2}+\left\|\delta_{\mu} \alpha_{l}\right\|_{2}^{2}=\left(\square_{\mu} \alpha_{l}, \alpha_{l}\right) \rightarrow 0, \text { as } l \rightarrow \infty .
$$

This shows that $\alpha \in D(\bar{\partial}) \cap D\left(\delta_{\mu}\right)$, and $\bar{\partial} \alpha=\delta_{\mu} \alpha=0 \mu$-a.e. Due to the hypoellipticity of $\square_{\mu}, \alpha$ is smooth, and hence, $\bar{\partial} \alpha=\delta_{\mu} \alpha=0$ pointwise.

A consequence of the celebrated Kohn's harmonic theory [10] is the Hodge decomposition of a vector bundle over a compact s.p.c. CR manifold. The corresponding result on a non-compact manifold, which is a consequence of Corollary 2.7, is the $L^{2}$-Hodge decomposition in the Main Theorem. Since the proof is similar to the case where $\eta \equiv 1$ and $E$ is trivial (e.g. [13]), we will omit the proof here.

\section{SERRE DUALITY}

In this section, we study Serre duality and complete the proof of the Main Theorem. Our method is to relate the operators on $E$ to those on $E^{*}$ via the weighted complex-conjugate Hodge star operator $\sharp_{\mu}$ (see e.g. [8], [6]). Together with results from the previous section, we obtain the Main Theorem. 
We start from the construction of $\sharp_{\mu}$. Let $*: \bigwedge^{k} T^{*} M \rightarrow \bigwedge^{2 n-1-k} T^{*} M$ be the Hodge star operator of $M$ with respect to $g$, which is uniquely determined by $g(* \alpha, \beta) d v=(n-1) ! \alpha \wedge \beta$, for $\alpha \in \bigwedge^{k} T^{*} M$ and $\beta \in \bigwedge^{2 n-1-k} T^{*} M . *$ is isometric and involutive, i.e. $g(* \alpha, * \beta)=g(\alpha, \beta)$ and $*^{2}=i d$, because $M$ is odd-dimensional. As the complexification of $*$ exchanges the set of holomorphic forms and the set of anti-holomorphic forms, the linear map $\sharp=\sharp_{M}:={ }^{-} \circ *$ satisfies (e.g. Lemma 7.1 [15]):

$$
\sharp\left(\Omega^{p, q}(M)\right)=\Omega^{n-p, n-(q+1)}(M) .
$$

We extend (3.1) to

Definition 3.1. Define

$$
\sharp_{\mu}: \Omega^{p, q}(E) \rightarrow \Omega^{n-p, n-(q+1)}\left(E^{*}\right)
$$

by

$$
\sharp_{\mu} \alpha:=\sum_{1 \leq i, j \leq r} \eta a_{j i}\left(\sharp \alpha^{i}\right) \otimes s^{j}, \text { for } \alpha \in \Omega^{p, q}(E),
$$

where $\alpha=\sum \alpha^{i} \otimes s_{i},\left\{s_{i}\right\}_{1 \leq i \leq r}$ is a local frame of $E,\left\{s^{i}\right\}$ is its dual frame of $E^{*}$, and $a_{i j}=\left\langle s_{i}, s_{j}\right\rangle_{E}$. Moreover, define $\sharp_{\mu}^{*}: \Omega^{n-p, n-(q+1)}\left(E^{*}\right) \rightarrow \Omega^{p, q}(E)$ by

$$
\sharp_{\mu}^{*} \phi:=\sum \eta^{-1} \bar{\alpha}^{i j}\left(\sharp \phi_{j}\right) \otimes s_{i},
$$

where $\phi=\sum \phi_{j} \otimes s^{j}$ and $a^{i j}=\left\langle s^{i}, s^{j}\right\rangle_{E^{*}}$, which is the entry of the inverse-matrix of $\left(a_{i j}\right)$. Here $E^{*}$ is furnished with the Hermitian fiber metric induced from $E$ :

$$
(\phi, \psi)_{E^{*}}=(\phi, \psi)_{\left(E^{*}, d \mu^{-}\right)}=\frac{1}{(n-1) !} \int \sum \phi_{i} \bar{\psi}_{j} a^{i j} d \mu^{-},
$$

where $\phi=\sum \phi_{j} \otimes s^{j}, \psi=\sum \psi_{i} \otimes s^{i}$, and $d \mu^{-}=\eta^{-1} d v$. For $\alpha \in \Omega^{p, q}(E)$ and $\phi \in \Omega^{s, t}\left(E^{*}\right)$, the product $\alpha \wedge \phi$ is defined by

$$
\alpha \wedge \phi:=\alpha^{i} \wedge \phi_{i} \in \Omega^{p+s, q+t}(M),
$$

where $\alpha=\sum \alpha^{i} \otimes s_{i}$ and $\phi=\sum \phi_{j} \otimes s^{j}$. The definition is well defined; i.e. it is independent of the choice of the frames. Similar to the Hodge star operator, it follows that

$$
(\alpha, \beta)_{E}=\frac{1}{(n-1) !} \int \alpha \wedge \sharp_{\mu} \beta, \text { for } \alpha, \beta \in \Omega(E),
$$

and

$$
(\phi, \psi)_{E^{*}}=\frac{1}{(n-1) !} \int \phi \wedge \sharp_{\mu}^{*} \psi, \text { for } \phi, \psi \in \Omega\left(E^{*}\right) .
$$

The operators $\sharp_{\mu}$ and $\sharp_{\mu}^{*}$ satisfy the following properties.

Proposition 3.2. It follows that

(i) $\sharp_{\mu}^{*} \sharp_{\mu}=i d_{\Omega(E)}$ and $\sharp_{\mu} \sharp_{\mu}^{*}=i d_{\Omega\left(E^{*}\right)}$;

(ii) $\left(\alpha, \sharp_{\mu}^{*} \phi\right)_{E}=\left(\sharp_{\mu} \alpha, \phi\right)_{E^{*}}$, for every $\alpha \in L^{2}(\Omega(E))$ and $\phi \in L^{2}\left(\Omega\left(E^{*}\right)\right)$.

Proof. (i) $\sharp_{\mu}^{*} \sharp_{\mu} \alpha=\sharp_{\mu}^{*}\left(\eta \bar{\alpha}_{j i} \overline{* \alpha^{i}} \otimes s^{j}\right)=\bar{\alpha}^{k j} \overline{*\left(a_{j i} * \bar{\alpha}^{i}\right)} \otimes s_{k}=\alpha$.

(ii) $\left(\alpha, \sharp_{\mu}^{*} \phi\right)_{E}=\left(\alpha, \sharp_{d v}^{*} \phi\right)_{(E, d v)}=\left(\sharp_{d v} \alpha, \phi\right)_{\left(E^{*}, d v\right)}=\left(\sharp_{\mu} \alpha, \phi\right)_{E^{*}}$.

We denote by $\bar{\partial}_{*}$ and $\delta_{\mu_{*}}$ the holomorphic structure of $L^{2}\left(\Omega\left(E^{*}\right)\right)$ and its formal adjoint, respectively. 
Lemma 3.3. It follows that

$$
\left(\alpha, \sharp_{\mu}^{*} \bar{\partial}_{* \sharp_{\mu}} \beta\right)_{E}=\left(\bar{\partial} \alpha,(-1)^{p+q+1} \beta\right)_{E},
$$

for $\alpha \in \Omega^{p, q}(E)$ and $\beta \in \Omega_{0}^{p, q+1}(E)$, and

$\left(3.4 k^{*}\right) \quad(-1)^{(p+q+1)}\left(\psi, \delta_{\mu_{*}} \phi\right)_{E^{*}}=\left(\psi, \sharp_{\mu} \bar{\partial} \sharp_{\mu}^{*} \phi\right)_{E^{*}}$

for $\phi \in \Omega^{p, q}\left(E^{*}\right)$ and $\psi \in \Omega_{0}^{p, q-1}\left(E^{*}\right)$.

Proof. Recall that the holomorphic structure $\bar{\partial}_{\wedge^{n}}$ coincides with the tangential Cauchy-Riemann operator $(-1)^{n} d^{\prime \prime}$ (e.g. Proposition 1.1 in [15]) defined as:

$$
d^{\prime \prime} f:=\left.d f\right|_{\bar{S}}, \text { for } f \in C(M) \text {. }
$$

Therefore, since $\sharp_{\mu} \beta \in \Omega^{n-p, n-(q+2)}\left(E^{*}\right)$ and $\alpha \wedge \sharp_{\mu} \beta \in \Omega_{0}^{n, n-2}(M)$,

$$
\begin{aligned}
0=(-1)^{n} \int d\left(\alpha \wedge \sharp_{\mu} \beta\right) & =(-1)^{n} \int d^{\prime \prime}\left(\alpha \wedge \sharp_{\mu} \beta\right) \\
& =\int \bar{\partial}_{\wedge^{n}}\left(\alpha \wedge \sharp_{\mu} \beta\right) \\
& =\int\left(\bar{\partial} \alpha \wedge \sharp_{\mu} \beta+(-1)^{p+q} \alpha \wedge \bar{\partial}_{* \sharp_{\mu}} \beta\right) \\
& =(\bar{\partial} \alpha, \beta)_{E}+\left(\alpha,(-1)^{p+q_{\sharp}^{*}} \bar{\partial}_{* \sharp_{\mu}} \beta\right)_{E} .
\end{aligned}
$$

We used (3.3) and Proposition 3.2 for the last step. We have (3.4).

Next, by taking into account that $\int d\left(\phi \wedge \sharp_{\mu}^{*} \psi\right)=0$, we can prove $\left(3.44^{*}\right)$ in a similar way.

We are now in a position to show:

Theorem 3.4 (Serre duality). It follows for every $0 \leq p, q \leq n-1$ that

$$
\sharp_{\mu}: \mathbb{H}^{p, q}(E) \cong \mathbb{H}^{n-p, n-(q+1)}\left(E^{*}\right) .
$$

Proof. Take $\alpha \in \mathbb{H}^{p, q}(E)$. Then $\alpha \in D(\bar{\partial}) \cap D(\delta), \bar{\partial} \alpha=0$ and $\delta_{\mu} \alpha=0$. By Proposition 3.2 and (3.4), for $\beta \in \Omega_{0}(E)$ we have that

$$
\begin{aligned}
\left(\delta_{\mu_{*}} \sharp_{\mu} \alpha, \sharp_{\mu} \beta\right)_{E^{*}} & =\left(\sharp_{\mu} \alpha, \bar{\partial}_{* \sharp_{\mu}} \beta\right)_{E^{*}} \\
& =\left(\alpha, \sharp_{\mu}^{*} \bar{\partial}_{*} \sharp_{\mu} \beta\right)_{E}=\left(\bar{\partial} \alpha,(-1)^{p+q} \beta\right)_{E}=0 .
\end{aligned}
$$

Since $\left\{\sharp_{\mu} \beta: \beta \in \Omega_{0}(E)\right\}$ is dense in $L^{2}\left(E^{*}\right)$ by Proposition 3.2, we have $\delta_{\mu_{*}} \sharp_{\mu} \alpha=0$. On the other hand, for $\phi \in \Omega_{0}\left(E^{*}\right)$, we have by Proposition 3.2 and $\left.(3.4)^{*}\right)$ that

$$
\begin{aligned}
(-1)^{p+q+1}\left(\bar{\partial}_{*} \sharp_{\mu} \alpha, \phi\right)_{E^{*}} & =(-1)^{p+q+1}\left(\sharp_{\mu} \alpha, \delta_{\mu_{*}} \phi\right)_{E^{*}} \\
& =\left(\sharp_{\mu} \alpha, \sharp_{\mu} \bar{\partial} \sharp_{\mu}^{*} \phi\right)_{E^{*}} \\
& =\left(\alpha, \bar{\partial} \sharp_{\mu}^{*} \phi\right)_{E} \\
& =\left(\delta_{\mu} \alpha, \sharp_{\mu}^{*} \phi\right)_{E}=0 .
\end{aligned}
$$

Therefore, $\bar{\partial}_{* \sharp_{\mu}} \alpha=0$, and we deduce that $\sharp_{\mu} \alpha \in \mathbb{H}^{n-p, n-(q+1)}\left(E^{*}\right)$.

The reverse implication can be shown by running the argumentation above from the bottom to the top. Now we obtain

$$
\sharp_{\mu}: \mathbb{H}^{p, q}(E) \cong \mathbb{H}^{n-p, n-(q+1)}\left(E^{*}\right),
$$

where $\sharp_{\mu}$ is a complex conjugate linear isomorphism. 


\section{EXAmples}

In this section, we present examples of non-compact s.p.c. CR manifolds with negligible boundary. Our argumentation relies on the following proposition.

\section{Proposition 4.1.}

(i) If $M$ is Riemannian complete, then it is complete with respect to $d_{C C}$.

(ii) If $M$ is complete, then $M$ has negligible boundary.

Proof. (i) Assume that $M$ is Riemannian complete, and let $\left\{x_{n}\right\}_{n>0} \subset M$ be a Cauchy sequence with respect to $\mathrm{d}_{C C}$. Since $\mathrm{d}_{g}$ has the alternative expression [12]

$$
\mathrm{d}_{g}(x, y)=\sup \left\{u(x)-u(y): u \in C^{\infty}(M),\|\nabla u\|_{L^{\infty}} \leq 1\right\},
$$

it follows that $\mathrm{d}_{g} \leq \mathrm{d}_{C C}$ by Equation (2.1). Hence, $\left\{x_{n}\right\}_{n>0}$ is a Cauchy sequence with respect to $\mathrm{d}_{g}$, and thus, the limit belongs to $M$, by the assumption. Since the topologies generated by $\mathrm{d}_{g}$ and $\mathrm{d}_{C C}$ are the same, we have the assertion.

(ii) Due to the fact that $M$ is complete, there exists a sequence $\left\{\chi_{l}\right\}_{l>0}$ of smooth functions with compact support such that $0 \leq \chi_{l} \leq 1, \chi_{l} \rightarrow 1$, and $\bar{\partial} \chi_{l} \rightarrow 0$ as $l \rightarrow \infty\left(\left[1\right.\right.$, [12]). For $\alpha \in D(\bar{\partial})$, set $\alpha_{l}=\chi_{l} \alpha$. Since $\alpha_{l}$ has compact support for every $l>0$,

$$
\left(\bar{\partial} \alpha_{l}, \beta\right)=\left(\alpha_{l}, \delta_{\mu} \beta\right) \rightarrow\left(\alpha, \delta_{\mu} \beta\right), \text { as } l \rightarrow \infty \text { for every } \beta \in D\left(\delta_{\mu}\right),
$$

where the left-hand side tends to $(\bar{\partial} \alpha, \beta)$ as $l \rightarrow \infty$.

The most fundamental example is

Example 4.2 (Heisenberg group). The Heisenberg group $\mathcal{H}(n)$ is

$$
\mathcal{H}(n)=\left\{(w, z) \in \mathbb{C}^{n} \times \mathbb{C}: \operatorname{Im} z=\|w\|^{2}\right\}
$$

with the group structure

$$
(w, z) \circ\left(w^{\prime}, z^{\prime}\right)=\left(w+w^{\prime}, z+z^{\prime}+2 \sqrt{-1} w \cdot w^{\prime}\right) .
$$

It is a quadratic CR submanifold (see e.g. 2]), whose defining function is

$$
f(w, z)=\operatorname{Im} z-\|w\|^{2} .
$$

Consider the following CR manifold which is CR-equivalent to $\mathcal{H}(n): \mathbb{C}^{n} \times \mathbb{R}$ with the contact form

$$
\theta=d t+2 \sum\left(x_{i} d y_{i}-y_{i} d x_{i}\right)
$$

Then since the orthonormal frame $\left\{X_{i}, Y_{i}\right\}$ of $P$ and the characteristic direction $\xi$ are given by

$$
X_{i}=\frac{1}{2} \frac{\partial}{\partial x_{i}}-y_{i} \frac{\partial}{\partial t}, Y_{i}=\frac{1}{2} \frac{\partial}{\partial y_{i}}+x_{i} \frac{\partial}{\partial t}, \text { and } \xi=\frac{\partial}{\partial t},
$$

no geodesics with respect to $g=-d \theta+\theta \otimes \theta$ reach $\infty$ in finite time. Due to the HopfRinow theorem, $\mathcal{H}(n)$ is Riemannian complete, and we conclude by Proposition 5.1 that $\mathcal{H}(n)$ is complete. Moreover, it was proved in [13] that $\mathbb{H}^{p, q}(E)=0$ for $0<q<n-1$ when $E$ is the trivial bundle over $\mathcal{H}(n)$.

Remark 4.3 ([13]). If $M$ has negligible boundary and additionally either

(1) the Ricci operator is positive on $\Omega^{p, q}$ or

(2) the Ricci operator is non-negative on $\Omega^{p, q}$ and $M$ has infinite volume, then $\mathbb{H}^{p, q}(E)=0$ for $0<q<n-1$ when $E$ is the trivial bundle over $M$. 
Example 4.4 (Sasakian space forms). There exist exactly three types of Riemannian complete simply connected Sasakian space forms: $S^{2 n+1}, \mathbb{R}^{2 n+1}$, and $D^{n} \times \mathbb{R}$, where $D \subset \mathbb{C}^{n}$ is a simply connected bounded domain with Kähler form $d \omega$ (e.g. [3]). The latter two space forms $\mathbb{R}^{2 n+1}$ and $D^{n} \times \mathbb{R}$ are non-compact and they have the contact form $d t-\sum_{i} y_{i} d x_{i}$ and $\omega+d t$, respectively. They have negligible boundary.

Example 4.5 (Spherical orbits). Let $O$ be the orbit of an $n^{2}$-dimensional automorphism in an $n$-dimensional non-homogeneous hyperbolic manifold. If $O$ is spherical, i.e. each point of $O$ has a neighbourhood which is CR-equivalent to an open set of $S^{2 n-1}$, then $O$ is CR-equivalent to one of the following hypersurfaces (e.g. 9]):

(1) A lens space $S^{2 n-1} / \mathbb{Z}_{m}$;

(2) $\sigma=\left\{(w, z) \in \mathbb{C}^{n-1} \times \mathbb{C}: \operatorname{Re} z=\|w\|^{2}\right\}$;

(3) $\sigma^{\prime}=\left\{(w, z) \in \mathbb{C}^{n-1} \times \mathbb{C}:|z|=\exp \|w\|^{2}\right\}$;

(4) $\omega=\left\{(w, z) \in \mathbb{C}^{n-1} \times \mathbb{C}:\|w\|^{2}+\exp (\operatorname{Re} z)=1\right\}$;

(5) $\omega_{\alpha}=\left\{(w, z) \in \mathbb{C}^{n-1} \times \mathbb{C}:\|w\|^{2}+|z|^{\alpha}=1, z \neq 0\right\}$, for some $\alpha>0$.

We are interested in $\sigma$ and $\sigma^{\prime}$ because they are non-compact. First we show that $\sigma$ is complete. Consider the map $\Phi: \mathcal{H}(n) \rightarrow \sigma$ defined as

$$
\Phi(w, z):=(w,-\sqrt{-1} z) .
$$

Clearly, $\Phi$ preserves the holomorphic structure. Moreover, since

$$
f_{\sigma} \Phi(w, z)=f_{H}(w, z)
$$

where $f_{\sigma}=\operatorname{Re} z-\|w\|^{2}$ is the defining function of $\sigma$, the contact structure is also preserved. Since $\mathcal{H}(n)$ is complete, so is $\sigma$.

Next we proceed to show that $\sigma^{\prime}$ is complete. The differential $d f_{\sigma^{\prime}}$ of the $\sigma^{\prime}$ s defining function $f_{\sigma^{\prime}}=|z|^{2}-\exp \|w\|^{2}$ is

$$
d f_{\sigma^{\prime}}=\frac{1}{2}\left(\frac{\bar{z}}{|z|} d z+\frac{z}{|z|} d \bar{z}\right)-\exp \|w\|^{2}\left(\sum\left(\bar{w}_{i} d w_{i}+w_{i} d \bar{w}_{i}\right)\right)
$$

and its pull-back $\Psi^{*} d f_{\sigma^{\prime}}$ by the covering map $\Psi: \sigma \rightarrow \sigma^{\prime}$, defined as $\Psi(w, z)=$ $(w, \exp z)$, is

$$
\begin{aligned}
& \frac{1}{2} \exp (\operatorname{Re} z)^{-1}\left(\exp \bar{z} \Psi^{*} d z+\exp z \Psi^{*} d \bar{z}\right)-\exp \|w\|^{2}\left(\sum\left(\bar{w}_{i} d w_{i}+w_{i} d \bar{w}_{i}\right)\right) \\
& \quad=\frac{1}{2} \exp (\operatorname{Re} z)^{-1} \exp (2 \operatorname{Re} z)(d z+d \bar{z})-\exp \|w\|^{2}\left(\sum\left(\bar{w}_{i} d w_{i}+w_{i} d \bar{w}_{i}\right)\right) \\
& \quad=\exp \|w\|^{2} d f_{\sigma},
\end{aligned}
$$

where we have used the fact that $\operatorname{Re} z=\|w\|^{2}$ for the last step. This shows that $\operatorname{ker}\left(\theta_{\sigma}\right)=\operatorname{ker}\left(\theta_{\sigma^{\prime}}\right)$ via $\Psi$. Moreover, since

$$
\Psi^{*} d \theta_{\sigma^{\prime}}=d \exp \|w\|^{2} \wedge \theta_{\sigma}+\exp \|w\|^{2} d \theta_{\sigma},
$$

where the first term on the right-hand side vanishes on $P$ and $\exp \|w\|^{2} \geq 1$, it follows that the distance associated to $\Psi^{*} d \theta_{\sigma^{\prime}}$ is not less than the one associated to $d \theta_{\sigma}$.

Thus, since these two distances generate the same topology and $\sigma$ is complete with respect to $d \theta_{\sigma}$, we may conclude that the distance associated to $\Psi^{*} d \theta_{\sigma^{\prime}}$ is complete by the same reason as in the proof of Proposition 4.1 .

We can also show that $\omega$ is complete, where the proof will appear in a forthcoming paper. 
Example 4.6. If $M$ is a compact s.p.c. CR manifold and $M^{\prime} \rightarrow M$ is an unbranched covering, then $M^{\prime}$ has negligible boundary.

Remark 4.7. Since the distance structure of $M$ is independent of the choice of the weight, all of those examples have negligible boundary with an arbitrary weight.

\section{Acknowledgments}

The authors wish to show their gratitude to Professor Nayatani and the referee for the stimulating discussion, for comments which made the paper more readable, and for providing us with an example of a CR manifold with negligible boundary.

\section{REFERENCES}

1. M. Biroli and U. Mosco, A Saint-Venant type principle for Dirichlet forms on discontinuous media, Annali di Matematica pura ed applicatata (1995), IV, 125-181. MR1378473 (97b:35082)

2. A. Boggess, CR manifolds and the tangential Cauchy-Riemann complex. Studies in Advanced Mathematics. CRC Press, Boca Raton, FL, 1991. MR1211412 (94e:32035)

3. D.E. Blair, Contact manifolds in Riemannian geometry. Lecture Notes in Mathematics, Vol. 509. Springer-Verlag, Berlin-New York, 1976. MR0467588 (57:7444)

4. W. Chow, Über Systeme von linearen partiellen Differentialgleichungen erster Ordnung. Math. Ann. 117, (1939). 98-105. MR0001880(1:313d)

5. G.B. Folland and J.J. Kohn, The Neumann problem for the Cauchy-Riemann complex. Annals of Mathematics Studies, No. 75. Princeton University Press, Princeton, NJ; University of Tokyo Press, Tokyo, 1972. MR0461588 (57:1573)

6. C.D. Hill and M. Nacinovich, Duality and distribution cohomology for CR manifolds, Ann. Scuola Norm. Sup. Pisa XXII (1995), 315-339. MR.1354910 (97g:32007)

7. L. Hörmander, Hypoelliptic second order differential equations. Acta Math. 119 (1967), 147171. MR0222474(36:5526)

8. M. Itoh and T. Saotome, The Serre duality for holomorphic vector bundles over a strongly pseudo-convex manifold, Tsukuba J. Math. 31 (2007), no. 1. 197-204. MR2337126

9. A. Isaev, Lectures on the automorphism groups of Kobayashi-Hyperbolic manifolds, Lecture Notes in Mathematics, Vol. 1902 (2007), VIII. MR2352328

10. J.J. Kohn, Harmonic integrals on strongly pseudoconvex manifolds, I, Ann. Math. 78 (1963), 112-148; II, ibid, 79 (1964), 450-472. MR0153030 (27:2999), MR0208200(34:8010)

11. J.J. Kohn and L. Nirenberg, Non-coercive boundary value problems, Comm. Pure Appl. Math. 18 (1965), 443-492. MR0181815 (31:6041)

12. J. Masamune, Essential self-adjointness of a sublaplacian via heat equation, Comm. Partial Differential Equations 30 (2005), no. 11, 1595-1609. MR2182306 (2006h:35035)

13. J. Masamune, Vanishing and conservativeness of harmonic forms of a non-compact CR manifold, Atti. Accad. Naz. Lincei Cl. Sci. Fis. Mat. Natur. Rend. Lincei (9) Mat. Appl. 19 (2008), no. 2, 79-102.

14. M. Reed and B. Simon, Methods of modern mathematical physics. II. Fourier analysis, selfadjointness. Academic Press, New York-London, 1975. MR0493420 (58:12429b)

15. N. Tanaka, A differential geometric study on strongly pseudoconvex CR manifolds, Kinokuniya Book Store Co., Ltd., Kyoto, 1975. MR0399517 (53:3361)

Institute of Mathematics, University of Tsukuba, 305-8751, Tsukuba, Japan

E-mail address: itohm@sakura.cc.tsukuba.ac.jp

Department of Mathematical Sciences, Worcester Polytechnic Institute, 100 Institute Road, Worcester, Massachusetts 01609-2280

E-mail address: masamune@wpi.edu

Graduate School of Pure and Applied Sciences, University of Tsukuba, 305-8571, TSUKUBA, JAPAN

E-mail address: tsaotome@math.tsukuba.ac.jp 